\title{
Generation of diffraction-free beams for applications in optical microlithography
}

\author{
M. Erdélyi, Z. L. Horváth, G. Szabó, and Zs. Bor \\ Department of Optics and Quantum Electronics, JATE University, H-6720 Szeged, Dóm tér 9, Hungary \\ F. K. Tittel ${ }^{\mathrm{a})}$ and J. R. Cavallaro \\ ECE Department, Rice University, MS 366, Houston, Texas 77005-1892
}

M. C. Smayling

Texas Instruments Inc., Stafford, Texas 77477

(Received 21 June 1996; accepted 13 December 1996)

\begin{abstract}
A new concept based on a Fabry-Pérot interferometer for the generation of nondiffracting Bessel beams is described and proposed for potential applications in microlithography such as the fabrication of small isolated patterns. It was experimentally demonstrated that the depth of focus can be increased by a factor of about 2 , and simultaneously the transverse resolution improved by a factor of 1.6, when using this technique to image contact holes. The properties of simultaneous imaging of two contact holes were also investigated. It was shown experimentally that, even in the most critical case (when the first diffraction rings overlap), undesirable interference effects between the adjacent contact holes can be eliminated by means of a phase shifting technique. (C) 1997 American Vacuum Society. [S0734-211X(97)00802-0]
\end{abstract}

\section{INTRODUCTION}

In 1987 Durnin $^{1}$ showed that the field described by

$$
E(r, z, t)=A \cdot J_{0}\left(k_{\perp} r\right) \cdot e^{i\left(k_{\|} z-\omega t\right)}
$$

is an exact solution of the wave equation

$$
\left(\nabla^{2}-\frac{1}{c^{2}} \frac{\partial^{2}}{\partial t^{2}}\right) E(r, z, t)=0,
$$

where $k_{\perp}^{2}+k_{\|}^{2}=\omega^{2} / c^{2}$, and $J_{0}$ is the zero order Bessel function of the first kind. The field described in Eq. (1) represents a nondiffracting beam because the transverse intensity distribution is independent of the propagation distance $z$. However, such an ideal beam cannot be realized experimentally over large values of $z$ and $r$, because it would represent a beam with infinite energy and spatial extent.

The first experimental arrangement to create a nearly nondiffracting Bessel beam was also suggested by Durnin. ${ }^{2}$ A circular slit (annular aperture) was placed at the focal plane of a lens. The slit was illuminated with collimated light. Each pointlike source along the slit was transformed by the lens into a plane wave whose wave vectors lie on the surface of a cone around the optical axis. The maximum $z$ value, for which the plane waves intersect and thus form a nondiffracting beam, was $Z_{\max }=D / \tan \theta$, where $D$ is the radius of the circular aperture, and $\theta$ is the angle that the $k$ vector makes with the $z$ axis $\left(\tan \theta=k_{\perp} / k_{\|}\right)$. The maximum range of the $J_{0}$ beam that could be realized experimentally was $85 \mathrm{~cm}$, and it could be fitted by numerical simulations. In 1992 Cox et al., ${ }^{3}$ based on the theoretical considerations by Indebetouw, ${ }^{4}$ produced a similar nondiffracting beam using a Fabry-Pérot interferometer. The ring system transmitted by the FabryPérot interferometer was collimated by a lens. An annular

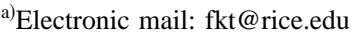

aperture placed at the focal plane of the lens transmitted only the first ring and blocked all the others. A second lens located after the spatial filter had the same role as in the experiment reported in Ref. 2 . It can be seen that the effective nondiffractive range using such a setup is $Z_{\max }=2 F d$, where $F$ and $d$ are the finesse and the thickness of the etalon, respectively. In fact, nondiffracting beams have also been generated by means of an axicon, ${ }^{5}$ holographic process, ${ }^{6,7}$ and a new type of laser cavity. ${ }^{8,9}$ It is worth noting that already a century ago ${ }^{10-15}$ it was recognized that the diffraction pattern of a very narrow annular aperture can be described by the $J_{0}$ function [Eq. (1)]. However, previous work paid less attention to the depth of focus and therefore did not describe such patterns in terms of nondiffracting beams. Only a few applications of nondiffracting beams have been reported so far. $^{16-18}$

This article reports an experimental demonstration of a new technique which is capable of enhancing the depth of focus (DOF) using nearly nondiffracting Bessel beams generated with a Fabry-Pérot interferometer. This technique can be a useful procedure in optical microlithography, especially for the fabrication of contact holes, because it significantly increases the depth of focus and simultaneously enhances the transverse resolution. Since in optical microlithography 1-2 $\mu \mathrm{m}$ DOF is sufficient, our purpose (in contrast with Refs. 2 and 3) is not to generate large-range constant-axial-intensity beams, but to increase the DOF only by a factor of about 2 . In Refs. 2 and 3 the generation of nearly nondiffracting Bessel beams was based on the imaging of an annulus placed in the focal plane of the lens. This annulus was created either with a circular slit or imaging and filtering the ring system of a Fabry-Pérot interferometer. In our experiments the FabryPérot interferometer is placed directly in front of the projection lens, and there is no other lens between the interferometer and projection lens, as described in Ref. 3. A detailed 


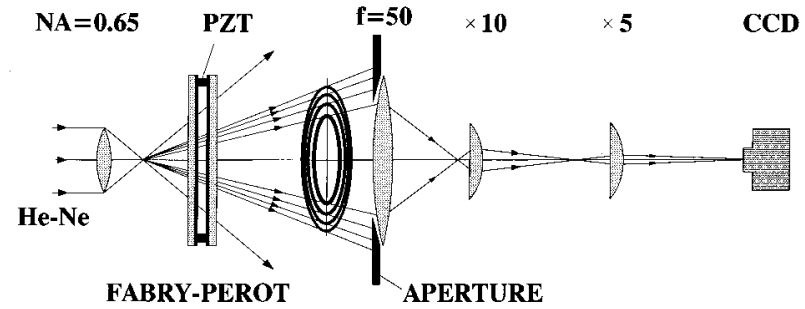

FIG. 1. Schematic diagram of the experimental setup. The aperture of the objective lens transmits the first Fabry-Pérot ring and blocks all other rings. The image was magnified by means of two microscope objectives and observed with a CCD camera.

theoretical treatment of the intensity distribution on the optical axis and in planes perpendicular to it, the transmitted energy and phase conditions - that supports the experimental data presented here-will be reported in a future publication. ${ }^{19}$

\section{EXPERIMENTAL DESCRIPTION OF TRANSFORMING A SINGLE POINTLIKE SOURCE INTO A NONDIFFRACTING BEAM}

The experimental arrangement is depicted in Fig. 1. A $\mathrm{He}-\mathrm{Ne}$ laser operating at $632.8 \mathrm{~nm}$ illuminates a microscope objective $(\mathrm{NA}=0.65)$. Due to the relatively high numerical aperture, the objective creates a nearly pointlike light source. This point source illuminates a scanning Fabry-Pérot interferometer (Tropel CL-100) which produces a concentric ring system behind the etalon. The aperture of an objective lens placed after the interferometer is adjusted so that it transmits only the first Fabry-Pérot ring (at the rim of the aperture) and blocks all the others. Due to multiple reflections in the interferometer (see Fig. 2), the real pointlike source $I_{0}$ creates several virtual images $\left(I_{1}, I_{2}, I_{3}, \ldots\right)$. The distance between these images is $2 d$, and the intensity ratio is $R^{2}$ between adjacent individual sources, where $R$ is the reflectivity

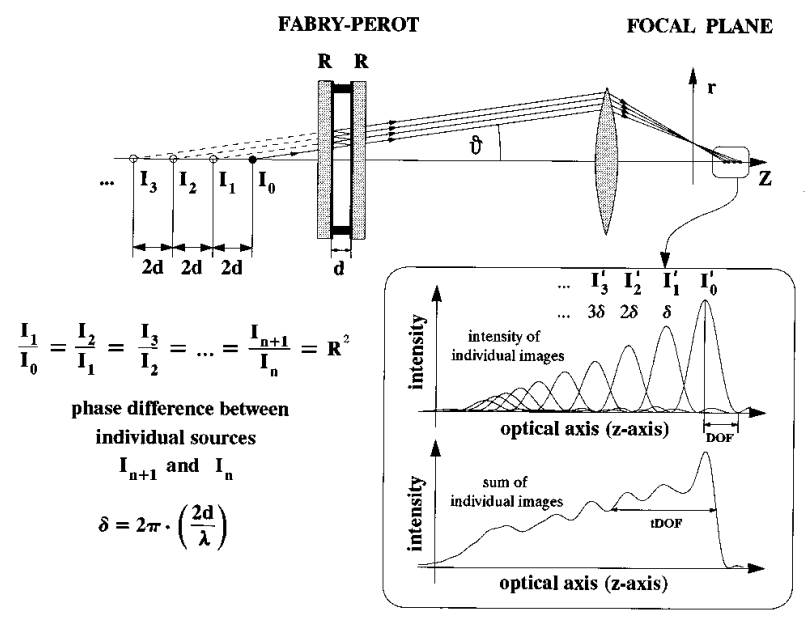

FIG. 2. The image produced by the objective lens is the superposition of the images $\left(I_{0}^{\prime}, I_{1}^{\prime}, I_{2}^{\prime}, \ldots\right)$ of the individual point sources. Therefore, $N$ (relative image density) is an important factor in determining the shape of the axial intensity distribution. of the Fabry-Pérot mirrors. The measured value of $R$ is $96.2 \%$. The distance between the virtual point sources can be changed easily as the separation of the interferometer is adjustable with a piezo translator. The relative phase difference $(\delta)$ between the adjacent point sources is related to $d$ by

$$
\delta=2 \pi \cdot \frac{2 d}{\lambda}
$$

The image produced by the objective is the superposition of the images of individual point sources. The distance between these points is $2 d M^{2}$, where $M^{2}$ is the longitudinal magnification of the objective lens. (The transverse magnification $M$ was measured using a USAF test target and was found to be $M=0.156$.) The image produced by the objective lens was magnified by two microscope objectives in tandem (with about 100 overall magnification) and monitored with a charge coupled device (CCD) camera (COHU 4810, horizontal center-to-center spacing of pixels: $23 \mu \mathrm{m})$. The first microscope objective was mounted on a precision translator, and thus the axial intensity distribution could also be examined.

It should be noted that our technique is similar to the FLEX method $^{20}$ where several focal planes are created at different positions along the optical axis, and exposures are made at each focal plane. Our technique, based on nondiffracting Bessel beams, creates multiple images of the mask onto the wafer but not requiring any mechanical shift. In this process, not the intensities but the electric fields are added. Hence, the final intensity distribution after the projection lens strongly depends on the phase difference between the individual pointlike sources.

\section{A. Axial intensity distribution of the image}

To describe the DOF enhancement of the new imaging technique it is necessary to define first the DOF of a conventional optical imaging system. The axial intensity distribution near the focus of a lens illuminated by a homogeneous beam is given by ${ }^{12}$

$$
I(u)=\frac{4 \pi^{2} D^{4}}{\lambda^{2} f^{2}} \cdot\left[\frac{\sin (1 / 4 u)}{1 / 4 u}\right]^{2},
$$

where $\lambda$ is the wavelength, $f$ is the focal length, and $u$ is a variable defined as

$$
u=\frac{2 \pi D^{2}}{\lambda f^{2}} z
$$

From Eq. (4) the depth of focus can be expressed as the distance between the first minimum and the main maximum

$$
\mathrm{DOF}^{*}=2 \frac{\lambda}{\mathrm{NA}^{2}}
$$

where $\mathrm{NA}=D / f$ is the numerical aperture of the lens. If the light source is not at infinity, the expression of $\mathrm{DOF}^{*}$ changes

$$
\mathrm{DOF}=\mathrm{DOF}^{*} \cdot(M+1)^{2} .
$$




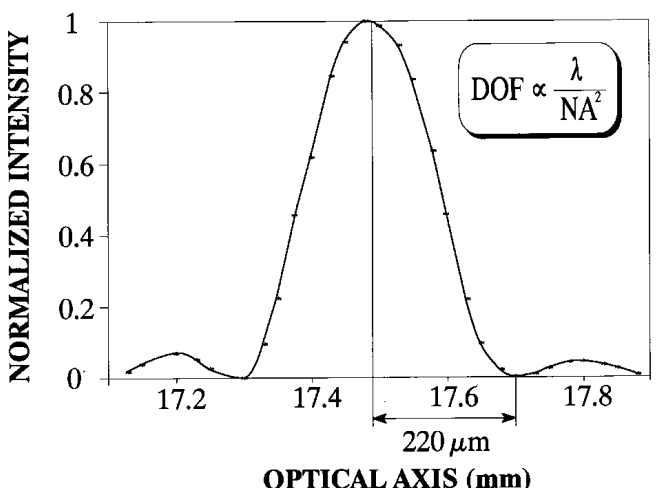

FIG. 3. The axial intensity distribution of a point source. The measured value of $220 \mu \mathrm{m}$ agrees well with the calculated $213 \mu \mathrm{m}$ value.
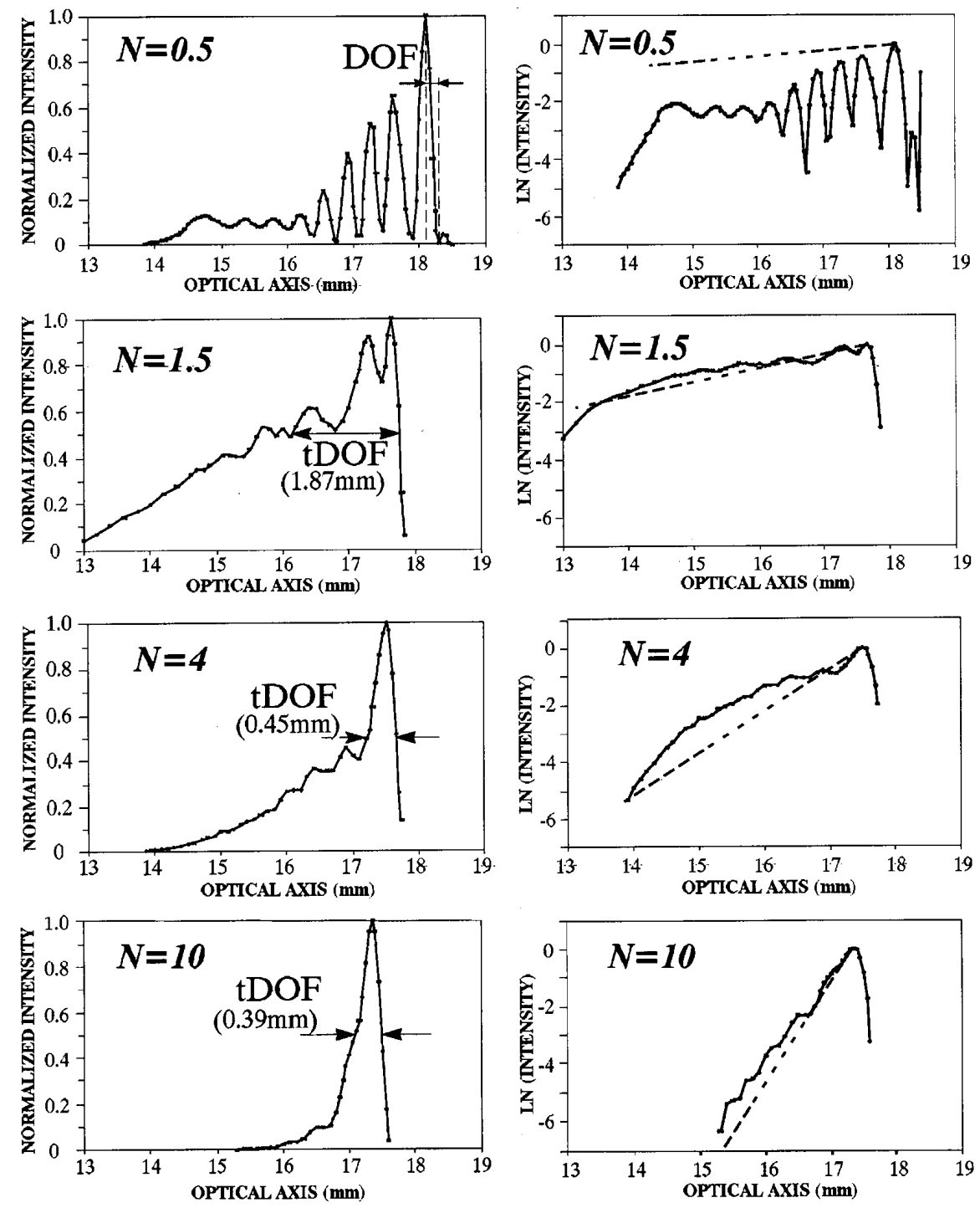

FIG. 4. Intensity distribution on the optical axis for different $N$ values. The position of the origin is arbitrary on the optical axis. (It is only determined by the initial position of the micrometer translator.) 
creases by increasing the $N$ ratio (reducing $d$ ). Four different experimental cases were studied $(N=0.5,1.5,4$, and 10).

Figure 4 shows the measured intensity distributions on the optical axis (linear and logarithmic scale) for the case of imaging a pointlike source. In the first case $(N=0.5)$, the distance between the image points is twice the DOF, and therefore the images can be observed separately. By decreasing the distance between the image points, the sharp peaks disappear and the intensity decreases faster on the optical axis. From a microlithographic point of view, oscillations in the intensity distribution are undesirable. By increasing the $N$ ratio, the curves become smoother and the oscillations disappear. In the last case $(N=10)$, no oscillations occur. Since in the case of superimposed images the first minimum is not zero, it is necessary to give a new definition of DOF denoted by $t \mathrm{DOF}$. The term $t \mathrm{DOF}$ is defined as the range where the intensity is larger than half of the main peak. The figures show normalized intensity, but in reality (due to the law of conservation of energy), by increasing the $N$ ratio, the intensity of the main peak increases. The measured values of $t \mathrm{DOF}$ are also depicted in the figures. The $N=4$ case is optimum for microlithographic applications. The oscillations have already disappeared, and the $t \mathrm{DOF}$ range is twice as large as without the Fabry-Pérot interferometer. The enhanced $t \mathrm{DOF}$ varies with the energy loss introduced by the Fabry-Pérot interferometer. Such an arrangement is very similar to an experiment in which an annular aperture is used as a pupil plane filter. It was shown previously by other authors $^{14}$ that using an annular aperture a $K$-fold gain in focal depth involves a $K$-fold loss of energy on transmission through the lens, and a $K^{2}$-fold loss of the intensity maximum in the focal plane. Due to such a model the loss of energy can be roughly estimated. For instance, in case of $N=4$ the depth of focus enhancement is $K=2.05$, and hence the predicted intensity maximum decreases to $23.8 \%$. The calculated intensity distribution after the projection lens will be discussed in Ref. 19.

The depth of focus enhancement can be roughly predicted using a very simple model. Combining the relations that describe the intensity difference is $1-R^{2}$ and the distance is $2 d M^{2}$ between two adjacent image points, we can write

$$
\ln (I)=-\frac{1-R^{2}}{2 d M^{2}} z
$$

The dashed lines in Fig. 4 depict this linear relationship. From Eq. (9) a simple estimate can be given for $t \mathrm{DOF}$ :

$$
t \mathrm{DOF}=K \cdot \mathrm{DOF} \quad \text { where } K=\frac{1}{N} \cdot \frac{\ln (2)}{1-R^{2}} .
$$

If $N=4$ the predicted increase of depth of focus is $K_{p} \approx 2.21$, while the measured value is about $K_{m} \approx 2.05$. The model, however is valid for a range of $N$. The two main limitations are: (1) The model does not consider that for the case $N<1$ the image points separate and thus the $t \mathrm{DOF}$ is approximately equal to DOF; (2) from Eq. (10) it can be seen that with increasing $N, K$ (and thus $t \mathrm{DOF}$ ) decreases and tends to zero. Fortunately, the range where the approximation is valid

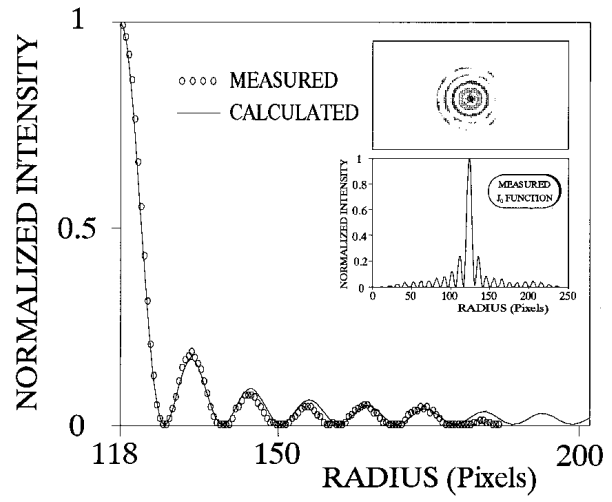

FIG. 5. The measured intensity distribution perpendicular to the optical axis is quasiequivalent to a zero order Bessel function. The solid line shows the fitted curve to the measured intensity distribution (depicted by circles).

(from $N=3.2$ to $N=7$ the difference between the measured and the predicted value is less than $20 \%$ ) coincides with the range that is applicable to microlithography.

\section{B. Intensity distribution in planes perpendicular to the optical axis}

The theoretically predicted intensity distribution in planes perpendicular to the optical axis is a $J_{0}$ function. ${ }^{19}$ The measured intensity distribution supports this prediction (see Fig. 5). The circles depict the measured intensity distribution, and the solid line shows the fitted $J_{0}$ Bessel function. The intensity of the high-order diffraction rings of the measured curve attenuates somewhat faster than the $J_{0}$ function. The small difference between the two curves can be explained by the finite size of the aperture. Each diffraction ring of the zero order Bessel function contains roughly equal amounts of energy, therefore, over an infinite plane the energy would be infinite. It is obvious that beams with infinite energy cannot be realized experimentally, and therefore the higher diffraction rings of the measured beam disappear. For instance, the intensity of the 12th and higher diffraction rings of the measured pattern depicted in the inset of Fig. 5 is effectively zero.

A comparison of the measured Bessel distribution and the Airy pattern shows (with good agreement of the theoretical description) that the full width at half-maximum (FWHM) of the Bessel beam is 1.6 times smaller than the (FWHM) of the Airy pattern (see Fig. 6). This decrease means an enhancement in the transverse resolution power, but it also has some disadvantages. The intensity of the successive diffraction rings is $16 \%, 9 \%, 6.2 \%, \ldots$, while the same values for the Airy pattern are $1.7 \%, 0.42 \%, 0.16 \%, \ldots .^{21}$ The interference of these relatively high intensity rings can cause problems if several points are imaged simultaneously. The overlapping of the first rings is critical for microlithography, as these rings have the highest intensity of all the diffraction rings.

\section{SIMULTANEOUS IMAGING OF TWO POINT SOURCES}

The previous section dealt with imaging one light source. However, in microlithography it is often necessary to expose 


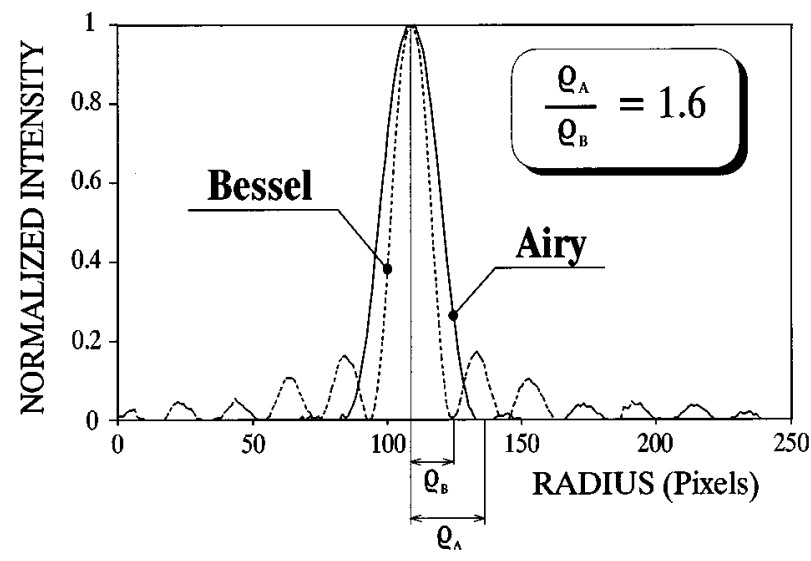

FIG. 6. Measured intensity distributions. The FWHM of the Bessel beam is 1.6 times smaller than the FWHM of the Airy pattern. This decrease means an enhancement in resolution power.

adjacent contact holes simultaneously. In this section we study the properties of imaging two contact holes, with special consideration to the most critical case, when the first diffraction rings overlap.

The distance and relative phase shift between the point sources are critical parameters. Recently, several phase shifting methods (PSM) have been proposed to enhance the DOF and the resolution power. ${ }^{22-25}$ The basic principle of PSM is that spatially coherent light transmitted through adjacent apertures is $180^{\circ}$ out of phase and therefore interferes destructively in the region between the images of the two apertures. Such a two point source generation method was used for which both the transverse distance and relative phase difference between the sources is adjustable.

The experimental setup is shown in Fig. 7. A Michelson interferometer was illuminated by a point source $P_{0}$ (a strongly focused $\mathrm{He}-\mathrm{Ne}$ laser). The interferometer created two virtual point sources $\left(P_{1}\right.$ and $\left.P_{2}\right)$ behind the mirror $M_{2}$.

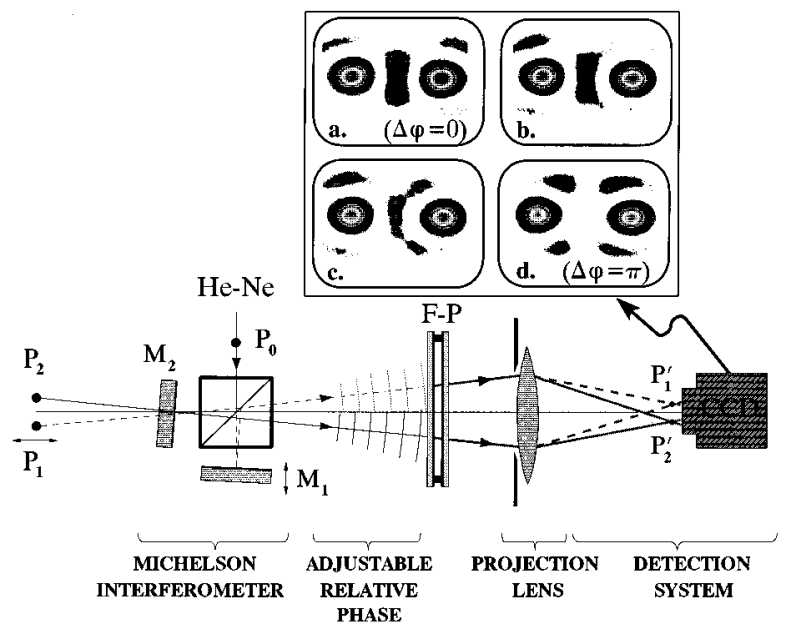

FIG. 7. Imaging of two coherent point sources formed by a Michelson interferometer. The transverse distance and the relative phase difference between the sources is adjustable by translating and tilting the mirrors. The inset shows the CCD images for different phase conditions.
By slighting tuning the mirrors $M_{1}$ and $M_{2}$, the relative transverse separation of $P_{1}$ and $P_{2}$ could be adjusted. The mirror $M_{1}$ was equipped with a piezoelectric transducer (PZT) translator, and thus the relative phase difference between the virtual $P_{1}$ and $P_{2}$ point sources could be arbitrarily adjusted. The other components (Fabry-Pérot interferometer, imaging lens, magnifying lens, and CCD detector) were the same as shown in Fig. 1.

Mirror $M_{1}$ and $M_{2}$ were adjusted so that the distance between the main peaks was twice the radius of the first diffraction ring (while they are at the same distance from the optical axis). This is the most critical case (as mentioned above), when the first rings overlap. The inset of Fig. 7 shows four different cases. In case $a$ there is constructive interference between the first diffraction rings (the phase shift is 0 ), and the intensity between the two main peaks can reach $64 \%$. However, if the relative phase shift is changed using a PZT element, then the intensity of the interference pattern between the main peaks decreases. Pictures $b$ and $c$ show intermediate cases when the phase shift is in the range of 0 to $\pi$. In case $d$ the phase difference is $\pi$, and, due to the destructive interference, the intensity maximum between the main peaks is zero. In this case the axial intensity distribution was also measured in a separate experiment. The result of this measurement showed that the axial intensity distribution of each spot $\left(P_{1}^{\prime}\right.$ or $\left.P_{2}^{\prime}\right)$ is independent of each other, which indicates that the $t \mathrm{DOF}$ does not change.

Although these experiments were performed with visible laser illumination, this method can be employed in 248 and $193 \mathrm{~nm}$ lithography. The predicted hole diameter at $193 \mathrm{~nm}$ is $0.24 \mu \mathrm{m}$ from Rayleigh's expression for resolution power. However, due to the resolution enhancement described in Sec. II B, contact holes with a diameter of $0.15 \mu \mathrm{m}$ should be feasible using an optical stepper with NA $=0.5$. Our experiments, where the effect of a PSM was simulated by a Michelson interferometer, show that, even in the most critical case, the undesirable effects of the interference of diffraction rings can be reduced with a phase shifting technique. Since the position of the pointlike source is not significant for the effect of the Fabry-Pérot interferometer, an off-axis hole can be imaged as long as the hole is inside the compensated field of the projection lens. Outside this area, lens aberrations deteriorate the image quality. Under this condition the technique is capable of imaging large field masks with off-axis holes.

\section{DISCUSSIONS AND CONCLUSIONS}

A novel concept for generating a diffraction-free beam has been proposed. The experimental results showed that this technique significantly increases both the DOF and the transverse resolution, which makes it attractive for applications in optical microlithography. This technique is especially effective when applied to small isolated patterns like contact holes. It was shown that even in the most critical case, undesirable interference effects between adjacent contact holes can be eliminated by means of a phase shifting mask. For more complex geometries, however, phase shifting may not 
be adequate; the imaging of real circuits needs further investigation. For possible application of this technique in a real optical stepper, the optimum parameters: thickness, reflectivity, and insertion point of the Fabry-Pérot interferometer have to be considered.

\section{ACKNOWLEDGMENTS}

This research was supported in part by Texas Instruments and the NSF under Grant Nos. DMI-9202639 and INT9020541, and by the QTKA Foundation of Hungary (No. T20910).

${ }^{1}$ J. Durnin, J. Opt. Soc. Am. 4, 651 (1987).

${ }^{2}$ J. Durnin, J. J. Miceli, Jr., and J. H. Eberly, Phys. Rev. Lett. 58, 1499 (1987).

${ }^{3}$ A. J. Cox and D. C. Dibble, J. Opt. Soc. Am. 9, 282 (1992).

${ }^{4}$ G. Indebetouw, J. Opt. Soc. Am. 6, 150 (1989).

${ }^{5}$ R. Arimoto, C. Saloma, T. Tanaka, and S. Kawata, Appl. Opt. 31, 6653 (1992).

${ }^{6}$ J. Turunen, A. Vasara, and A. T. Friberg, Appl. Opt. 27, 3959 (1988).

${ }^{7}$ A. J. Cox and D. C. Dibble, Appl. Opt. 30, 1330 (1991).

${ }^{8}$ J. K. Jabczynski, Opt. Commun. 77, 292 (1990).

${ }^{9}$ K. Uehara and H. Kikuchi, Appl. Phys. B 48, 125 (1989).
${ }^{10}$ G. B. Airy, Philos. Mag. 18, 1 (1841).

${ }^{11}$ G. S. Steward, Philos. Trans. R. Soc. London Ser. A 225, 131 (1926).

${ }^{12}$ E. H. Linfoot and E. Wolf, Proc. Phys. Soc. London Sec. B 66, 145 (1953).

${ }^{13}$ C. A. Taylor and B. J. Thompson, J. Opt. Soc. Am. 48, 844 (1958).

${ }^{14}$ W. T. Welford, J. Opt. Soc. Am. 50, 749 (1960).

${ }^{15}$ C. W. McCutchien, J. Opt. Soc. Am. 54, 240 (1964).

${ }^{16}$ M. R. LaPointe, Proc. SPIE 1527, 258 (1991).

${ }^{17}$ G. Häusler and W. Heckel, Appl. Opt. 27, 5165 (1988).

${ }^{18}$ F. Schellenberg, M. D. Levenson, and P. J. Brock, Proc. SPIE 1604, 274 (1991).

${ }^{19}$ Z. L. Horvath, M. Erdelyi, G. Szabo, Zs. Bor, F. K. Tittel, and J. R. Cavallaro (unpublished).

${ }^{20}$ H. Fukuda, N. Hasegawa, and S. Okazaki, J. Vac. Sci. Technol. B 7, 667 (1989).

${ }^{21}$ M. Born and E. Wolf, Principle of Optics, 6th ed. (Pergamon, New York, 1980), p. 397.

${ }^{22}$ M. D. Levenson, N. S. Viswanathan, and R. A. Simpson, IEEE Trans. Electron Devices ED-29, 1828 (1982); M. D. Levenson, Jpn. J. Appl. Phys. 1 33, 6765 (1994).

${ }^{23}$ M. Shibuya, Japanese Patent No. Showa 62-50811 (30 September 1980).

${ }^{24}$ H. Watanabe and Y. Todokoro, J. Vac. Sci. Technol. B 11, 2669 (1993).

${ }^{25}$ M. Erdélyi, Zs. Bor, J. R. Cavallaro, G. Szabó, W. L. Wilson, C. Sengupta, M. C. Smayling, and F. K. Tittel, Jpn. J. Appl. Phys. 1 34, L1629 (1995). 